\title{
Emergence of fluoroquinolone resistance among drug resistant tuberculosis patients at a tertiary care facility in Karachi, Pakistan
}

Syed Mohammad Asad Zaidi', Abdul Haseeb², Shifa Salman Habib", Amyn Malik', Saira Khowaja', Nausheen SaifUllah ${ }^{2}$ and Nadeem Rizvi ${ }^{2}$

\begin{abstract}
Background: Pakistan is classified as one of the high multi-drug resistant tuberculosis (MDR-TB) burden countries. A poorly regulated private sector, over-prescription of antibiotics and self-medication has led to augmented rates of drug-resistance in the country. Pakistan's first national anti-tuberculosis drug resistance survey identified high prevalence of fluoroquinolone resistance among MDR-TB patients. Further institutional evidence of fluoroquinolone drugresistance can support re-evaluation of treatment regimens as well as invigorate efforts to control antibiotic resistance in the country.
\end{abstract}

Findings: In this study, data for drug-susceptibility testing (DST) was retrospectively analyzed for a total of 133 patients receiving MDR-TB treatment at the Chest Department of Jinnah Postgraduate Medical Center, Karachi, Pakistan. Frequency analyses for resistance patterns was carried out and association of fluoroquinolone (ofloxacin) resistance with demographics and past TB treatment category were assessed. Within first-line drugs, resistance to isoniazid was detected in $97.7 \%$ of cases, followed by rifampicin (96.9\%), pyrazinamide (86.4\%), ethambutol (69.2\%) and streptomycin (64.6\%). Within second-line drugs, ofloxacin resistance was detected in $34.6 \%$ of cases. Resistance to ethionamide and amikacin was $2.3 \%$ and $1.6 \%$, respectively. Combined resistance of oflaxacin and isoniazid was detected in $33.9 \%$ of cases. Age, gender and past TB treatment category were not significantly associated with resistance to ofloxacin.

Conclusion: Fluoroquinolone resistance was observed in an alarmingly high proportion of MDR-TB cases. Our results suggest caution in their use for empirical management of MDR-TB cases and recommended treatment regimens for MDR-TB may require re-evaluation. Greater engagement of private providers and stringent pharmacy regulations are urgently required.

Keywords: Multi-drug resistant tuberculosis, Fluoroquinolone resistance, Pakistan

\section{Findings}

\section{Background}

Globally, emerging drug resistance poses a serious threat to the control of tuberculosis (TB) [1]. In 2012, the World Health Organization (WHO) estimated 450,000 (range

\footnotetext{
*Correspondence: shifa.habib@irdresearch.org

${ }^{1}$ Interactive Research \& Development, Suite 508, Ibrahim Trade Tower, Main Shahrah-e-Faisal, Karachi, Pakistan

Full list of author information is available at the end of the article
}

300,000-600,000) new cases of multidrug-resistant TB (MDR-TB) in the world; $3.6 \%$ of newly diagnosed TB cases, causing an estimated 170,000 (range 102,000$142,000)$ deaths [2]. Pakistan contributes to $69 \%$ of the MDR-TB burden in the Eastern Mediterranean Region (EMRO), with an estimated 11,000-29,000 cases occurring in 2012 [2]. Over the past few years the country has also reported an increasing trend of Extensively Drug Resistance disease (XDR-TB) that includes resistance 
to fluoroquinolones and at least one other second-line injectable, in addition to MDR-TB [3].

Fluoroquinolone resistance is of particular public health importance as it is a widely prescribed antibiotic for treatment of common respiratory tract infections. There is a rampant and poorly regulated private healthcare sector in Pakistan, where over-prescription of fluoroquinolones by general practitioners, over-the-counter sales at private pharmacies and self-medication by patients occur commonly [4]. Recently published findings from the first national anti-tuberculosis drug resistance survey of Pakistan identified fluoroquinolone resistance of $26.6 \%$ (95\% CI 18.0-36.7) among a total sample of 96 MDR-TB cases [5]. Evidence suggests that occurrence of fluoroquinolone resistance is on the rise, with a study reporting increase in its prevalence from $17.4 \%$ in 2005 , to $53.9 \%$ in 2014 among MDR-TB patients [6].

Fluoroquinolones are the mainstay of treatment of MDR-TB and resistance has been associated with poor treatment outcomes for MDR-TB patients [6]. Rising fluoroquinolone resistance is, therefore, of serious concern for the management of MDR-TB patients, particularly at a time when Pakistan is scaling-up its Programmatic Management of Drug-resistant TB (PMDT) program [7]. Scale-up of Xpert MTB/RIF testing in the country has significantly facilitated diagnosis of MDR-TB since resistance to rifampicin can be identified at the time of diagnosis [8]. However, patients initially require empirical treatment as drug-susceptibility testing (DST) results to fluoroquinolones and other second-line drugs require 6-8 weeks by conventional methods. A lack of clinical response to medication can be alarming for both patients and providers [9]. In the setting of increasing resistance to fluoroquinolones, current combinations of empirical therapy used by MDR-TB programs may require revaluation. Further institutional evidence of fluoroquinolone drug-resistance among MDR-TB patients can support such recommendations as well as boost efforts to control antibiotic resistance in the country. This study describes the drug-resistance patterns of microbial isolates from MDR-TB suspects from a tertiary-care facility in Karachi, Pakistan from 2009 to 2012.

\section{Methods}

We analyzed de-identified, retrospective data of patients receiving MDR-TB treatment at Jinnah Postgraduate Medical Center, the largest public-sector hospital in Pakistan's southern Sind province, between 2009 and 2012. All patients enrolled on MDR-TB program had a history of TB treatment and were included in the analysis using convenience sampling. Sputum was obtained from the patients prior to MDR-TB treatment initiation and DST of Mycobacterium tuberculosis isolates was carried out at the Aga Khan University clinical laboratory, a technical partnering facility for the National TB Program (NTP). Demographic and clinical histories of the patients were obtained from the hospital records. Treatment history was categorized as prior history of Category I TB treatment (four-drug anti-TB treatment regimen) and Category II (four-drug anti-TB treatment regimen + streptomycin). Frequency analyses for resistance patterns and demographic variables were carried out. The association of ofloxacin (a second-generation fluoroquinolone) resistance with age was investigated using Student's t test. Chi squared tests were conducted to investigate the association of ofloxacin resistance with gender and treatment history. Statistical analyses were carried out using Stata/IC 12.0 (Stata Corp, College Station, TX, USA).

\section{Results}

A total of 133 patients receiving MDR-TB treatment were included in the study. Median age of the patients was 16 (IQR 10-26) and 51.1\% were male (Table 1).

Within first-line drugs, resistance to isoniazid was reported in $97.7 \%$ of cases followed by rifampicin (96.9\%), pyrazinamide $(86.4 \%)$, ethambutol $(69.2 \%)$ and streptomycin (64.6\%) (Table 2).

Within second-line drugs, ofloxacin resistance was observed in $34.6 \%$ of cases. Resistance to ethionamide and amikacin was 2.3 and $1.6 \%$, respectively (Table 2). Combined resistance to ofloxacin and isoniazid was reported in $33.9 \%$ of cases (Table 2). Ofloxacin resistance was more commonly observed in patients with history of Category I treatment (54.3\%) compared to Category II (40.7\%), however, the difference was not statistically significant ( $p$ value 0.26 ). Age ( $p$ value 0.92 ) and gender ( $p$ value 0.85 ) were not significantly associated with ofloxacin resistance. A total of 3 cases were found to have XDR-TB.

Table 1 Demographic characteristics of MDR-TB patients at Jinnah Postgraduate Medical Center, Karachi, Pakistan (2009-2012)

\begin{tabular}{lcc}
\hline & $\mathbf{n}$ & $\mathbf{( \% )}$ \\
\hline Age (years) & 10 & \\
$9-14$ & 71 & $(8)$ \\
$15-29$ & 33 & $(54)$ \\
$30-44$ & 19 & $(25)$ \\
$45<$ & & $(14)$ \\
Gender & 68 & \\
Male & 65 & $(51.1)$ \\
Female & 133 & $(48.9)$ \\
Total & & \\
\hline
\end{tabular}


Table 2 Resistance to first and second-line anti-tuberculous medication among MDR-TB patients at Jinnah Postgraduate Medical Center, Karachi, Pakistan (2009-2012)

Number of cases with resistance

n (\%)

Any resistance (first line drugs)
Isoniazid (H)
Rifampicin (R)
Pyrazinamide ( $\mathrm{C}$ )
Ethambutol (E)
Streptomycin ( $\mathrm{S}$ )
Any resistance (second line drugs)
Kanamycin (Km)
Amikacin (Am)
Capreomycin (Cm)
Ofloxacin (Ofx)
Ethionamide (Eto)
Cycloserine (Cs)
Combined resistance of H with other drugs
$\mathrm{H}+\mathrm{Z}$
$\mathrm{H}+\mathrm{E}$
$\mathrm{H}+\mathrm{S}$
$\mathrm{H}+\mathrm{Z}+\mathrm{E}$
$\mathrm{H}+\mathrm{Z}+\mathrm{E}+\mathrm{S}$
$\mathrm{H}+\mathrm{Ofx}$

\section{0}

129

115

92

86

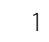

2

2

49

3

1
(97.7)

(96.9)

(86.4)

(69.2)

(64.6)

$(0.8)$

$(1.5)$

(1.5)

(34.6)

(2.3)

$(64.7)$

$(67.7)$

(50.4)

(33.9)

\section{Discussion}

Our results support findings from previously reported studies and the national anti-TB drug resistance survey, highlighting growing resistance to fluoroquinolones in Pakistan. This is the first study describing institutional evidence from an MDR-TB treatment facility of the high prevalence of fluoroquinolone resistance among MDR-TB cases. Resistance was not associated with age, gender or history of TB treatment, suggesting that resistance may be widespread within this patient population. These findings raise concerns for the outcomes of MDR-TB patients and for empirical treatment, following rifampicin resistance identification through an Xpert MTB/RIF assay. Recommended treatment regimens for MDR-TB may therefore require revaluation for improved treatment outcomes and to curtail development of further drug-resistance.

Given the above scenario, several public health policy measures and programmatic interventions can be of benefit. Firstly, the use of antibiotics, particularly fluoroquinolones needs to be strongly regulated. This is likely to be a major challenge, given Pakistan's large and fragmented private healthcare sector. A regulation restricting over-the-counter sale of antibiotics also needs to be introduced, the practical implementation of which could be difficult. Secondly, strategies to engage with the private-sector providers more comprehensively need to be explored. Recent initiatives proposed by the National TB Control program are encouraging [10]. Under novel public-private mix approaches, local networks and partnerships can be created where private providers receive medication through provincial TB control programs for patients and in turn, help to increase case-notification. Greater efforts should be in place to collaborate with general practitioners, especially with family practitioners operating in small clinics in urban areas. Trainings should be conducted on NTP guidelines for TB casedetection and treatment as well as on more judicious use of antibiotics for treatment of common infections, with a special emphasis on restricting use of fluoroquinolones. Medical associations, including those representing infectious disease specialists, pulmonologists as well as pharmacists need to be play a more active role in communicating this message to providers and to the public. Finally, MDR-TB programs will require greater efforts to improve case-holding and ensuring treatment completion for enrolled patients, as PMDT services expand in the country. Community-based solutions and behavioral economics approaches such as the use of food-baskets and other incentives for treatment compliance may be of benefit [11]. Early case-detection through scale-up of Xpert MTB/RIF testing may help improve treatment outcomes as well as limit transmission of resistant strains. This study was limited by the absence of DST results for third and fourth generation fluoroquinolones for assessing cross-resistance with ofloxacin of $M$. tuberculosis isolates. In addition, limited patient history was available, such as prior history of quinolone use, to further investigate risk factors and clinical correlates to fluoroquinolone resistance. We recommend further studies to evaluate MDR-TB treatment outcomes and their association with fluoroquinolone resistance among DR-TB patients.

\section{Conclusions}

Fluoroquinolone resistance was observed in an alarmingly high proportion of MDR-TB cases. Our results suggest caution in their use for empirical management of MDR-TB cases (particularly second-generation) and therefore recommended treatment regimens for MDRTB may require revaluation. Engaging private sector providers in TB case-detection and stringent pharmacy regulations are required to prevent increased resistance to fluoroquinolones in Pakistan.

\section{Abbreviations}

TB: tuberculosis; MDR-TB: multi-drug resistant tuberculosis; EMRO: Eastern Mediterranean Region; XDR-TB: extensively drug resistant tuberculosis; PMDT: Programmatic Management of Drug-resistant; DST: drug Susceptibility 
testing; NTP: National Tuberculosis Control program; MTB/RIF: mycobacterium tuberculosis/rifampicin.

\section{Authors' contributions}

$\mathrm{NR}, \mathrm{AH}$ and NS were involved in conception of the study, finalizing the study design and conducting data collection. SMAZ was involved in conducting the literature review, data analysis, data interpretation and drafting the manuscript. SSH, AM, and SK reviewed the drafts critically and finalized the manuscript. All authors read and approved the final manuscript.

\section{Author details}

${ }^{1}$ Interactive Research \& Development, Suite 508, Ibrahim Trade Tower, Main Shahrah-e-Faisal, Karachi, Pakistan. ${ }^{2}$ Department of Chest Medicine, Jinnah Post Graduate Medical Centre, Rafiqi H J Rd, Karachi, Pakistan.

\section{Acknowledgements}

Not applicable.

\section{Competing interests}

The authors declare that they have no competing interests.

\section{Availability of data and materials}

The datasets analysed during the current study are not publicly available, as they contain patient information, the overall ownership of which lies with the public sector hospital where the patients were treated and with the Provincial Tuberculosis Program. The data can be made available by the corresponding author on reasonable request, after necessary permissions from the hospital and the public sector authorities.

\section{Ethics approval and consent to participate}

For this retrospective analysis, an exemption for ethical review was obtained from the Ethics Review Committee at Jinnah Postgraduate Medical Center. Consent to participate was not applicable, as de-identified retrospective data was used for our analysis.

\section{Publisher's Note}

Springer Nature remains neutral with regard to jurisdictional claims in published maps and institutional affiliations.

Received: 15 August 2016 Accepted: 17 July 2017

Published online: 25 July 2017

\section{References}

1. Organization WH. Global tuberculosis control: surveillance, planning, financing: World Health Organization; 2007.

2. Organization WH. The Burden of Disease Caused by TB. Geneva: World Health Organization; 2013.

3. Hasan R, Jabeen K, Ali A, Rafiq Y, Laiq R, Malik B, et al. Extensively drugresistant tuberculosis, Pakistan. Emerg Infect Dis. 2010;16(9):1473-5.

4. Jabeen K, Shakoor S, Chishti S, Ayaz A, Hasan R. Fluoroquinolone-resistant Mycobacterium tuberculosis, Pakistan, 2005-2009. Emerg Infect Dis. 2011;17(3):566.

5. Tahseen S, Qadeer E, Khanzada FM, Rizvi AH, Dean A, Van Deun A, Zignol M. Use of $\mathrm{Xpert}^{\mathbb{B}} \mathrm{MTB} / \mathrm{RIF}$ assay in the first national anti-tuberculosis drug resistance survey in Pakistan. Int J Tuberc Lung Dis. 2016;20(4):448-55.

6. Ahmad N, Khan AH, Sulaiman SA, Javaid A. Fluoroquinolones resistance in multidrug-resistant tuberculosis in Pakistan and suitability of guidelines recommended standardized regimen. Int J Mycobacteriol. 2015;3(4):258-9.

7. The National Tuberculosis Control Program-Pakistan: http://www.ntp.gov. pk/. Accessed 20 June 2016

8. Creswell J, Codlin AJ, Andre E, Micek MA, Bedru A, Carter EJ, et al. Results from early programmatic implementation of Xpert MTB/RIF testing in nine countries. BMC Infect Dis. 2014;14:2.

9. Guidelines for the Programmatic Management of Drug-Resistant Tuberculosis: 2011 Update. WHO Guidelines Approved by the Guidelines Review Committee. Geneva, 2011.

10. Chughtai AA, Qadeer E, Khan W, Hadi H, Memon IA. Estimation of the contribution of private providers in tuberculosis case notification and treatment outcome in Pakistan. East Mediterr Health J. 2013;19(3):213-8.

11. Khan AJ, Khowaja S, Khan FS, Qazi F, Lotia I, Habib A, et al. Engaging the private sector to increase tuberculosis case detection: an impact evaluation study. Lancet Infect Dis. 2012;12(8):608-16.

\section{Submit your next manuscript to BioMed Central and we will help you at every step:}

- We accept pre-submission inquiries

- Our selector tool helps you to find the most relevant journal

- We provide round the clock customer support

- Convenient online submission

- Thorough peer review

- Inclusion in PubMed and all major indexing services

- Maximum visibility for your research

Submit your manuscript at www.biomedcentral.com/submit
() Biomed Central 